\title{
仰卧港腹測試法：測定腹肌耐力的新方法
}

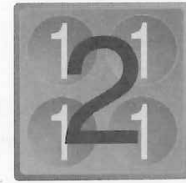

\section{Curl-Up Test: A New Abdominal Muscular Endurance Test}

\author{
鍾伯光博士 \\ 香港浸會大學體育系副教授 \\ Dr. Chung Pak-kwong \\ Associate Professor, Department of Physical Education \\ Hong Kong Baptist University
}

Sit-up is a popular exercise for both athletes and health fitness participants. It is almost universally accepted as an effective training method of abdominal strength and endurance. The 1-minute timed sit-up (modified sit-up) test, modified from the straight-legged sit-up with hands locked behind the head, is widely used in the health fitness testing such as Physical Best of AAHPERD, National Children and Youth Fitness Study (NCYFS \& NCYFS II) of the United States Department of Health and Human Services, and Survey of Health Related Fitness \& Lifestyle of Working Population Aged 20-39 in Hong Kong conducted by Hong Kong Physical Fitness Association etc. Although the modified sit-up was assumed to be a safer test than the straightlegged sit-up, researchers are still not satisfied with it. Are there any problems with sit-up test? Is the curl-up test developed by Robertson and Magnusdottir (1987) safer and more valid and reliable than the modified sit-up test? All such queries are being discussed in this article.

\section{- : 引言}

運動科學及醫學專家 (AAHPERD, 1988; Corbin, 1987; Falls, Baylor, \& Dishman, 1980; Seefeldt \& vogel, 1986) 都一致認為良好的健康體適能 (Health-Related Physical Fitness) 有助個人應付日常工作的負荷和壓力, 提供足夠精力去參與餘閒活動, 承受意外或突發事情, 以及預防和對抗一些與運動不足有關的疾病 (Hypokinetic Diseases) (Kraus \& Raab,1961)，如冠心病、高血壓、糖尿病、肌肉及骨骼等毛病。

希活 (Heyward, 1991) 認為健康體適能應包括心肺耐力 (Cardiorespiratory Endurance) 、肌力及肌耐力 (Muscular Strength and Endurance) 、柔軟度 (flexibility) 、身體組合 (Body Composition) 和神經肌肉的放鬆 能力 (Neuromuscular Relaxation) 等五項要素。其中, 下背痛 (Low Back Pain) 更被認為跟腹部肌肉耐力不 足有關 (Macfarlane, 1993; Plowman, 1992) 。因此，腹部肌肉的鉎鍊，經常受到健體運動者的重視。

在鍛鍊的同時, 為了能夠了解健體參與者的體能狀沉, 從而設定其運動計劃的內容, 專家們針對各項體適 能要素而設計出不同的測試方法。在肌耐力方面, 腹肌耐力被視為重要的測試對像。由於獲得眾多研究者和 專家 (Flint \& Gudgell, 1965; Gutin \& Lipetz, 1971 ; Noble, 1975; Ricci, Marchetti, \& Figura, 1981 ; Walters \& Partridge, 1975) 的支持, 仰臥起坐 (Sit-up) 被視為一項促進腹肌耐力的運動。

然而，仰臥起坐的動作卻一直受着研究者 (DeLacerda, 1978；Kelley, 1982；Kendall, 1965； Sinaki, \& Mikkelsen, 1984) 質疑。他們提出的問題是仰臥起坐, 特別是當雙腳被固定時, 會增加稳屈肌群 (Hip Flexors) 的參與程度, 令腰椎過度受壓而可能造成下背痛。鑑於這個原因, 仰臥起坐亦由早期的直膝姿勢改變為曲膝 姿勢, 藉此減少下背在動作進行時承受的壓力。同時, 雙手抱在頭後的手部動作亦被轉變為雙手交叉置於胸 腹上。像這種修改式仰臥起坐 (Modified Sit-up) 在八十年代間廣泛被用在健康體適能的測試項目中，如美國 健康, 體育、康樂和舞蹈聯盟 (American Alliance for Health, Physical Education, Recreation and Dance) 採用 的測試 (Physical Best) (AAHPERD, 1980)，美國健康及人類服務部 (United States Department of Health and Human Services - USDHHS) 統籌設計的國際兒童及青少年體適能研究 (National Children and Youth Fitness Study -NCYFS) 採用的測試 NGYFS (USDHHS, 1985) 和 NCYFS II (USDHHS, 1987)，以及香港體適能總 會進行了的體適能推廣計劃中所用的測試 (Hong Kong Physical Fitness Association, 1993) 等。

雖然如此，仰臥起坐仍然存在問題，部份專家 (Gutin \& Lipetz, 1971; Kelley, 1982； Macfarlane, 1993; Robertson \& Magnusdottir, 1987; Vincent \& Britten, 1980) 更建議利用仰卧捲腹 (Curl-up) 來取代仰卧起坐。 究竟仰臥起坐的問題在那裡? 仰臥捲腹在鈠鍊腹部肌肉比仰臥起坐更加安全和有效嗎? 仰臥捲腹能否準確和 可信地用在腹肌耐力的測試? 這些都是本文探討的範圍。

\section{二・仰歐起坐的問題在那裡?}

傳統的直膝及把雙手抱在頭後的仰臥起坐 (圖一)，因被認為對腰椎 (直滕坐起動作) 和頸椎 (雙手用力 把頭拉向上和前的動作) 造成壓力, 可能引致這兩部份受傷。因此, 曲膝及把雙手交叉置於胸腹的修改式仰 臥起坐便起而代之，但這種經改良的仰卧起坐，仍鼓勵參與者進行全幅度 (Full range of motion) 的坐起動 


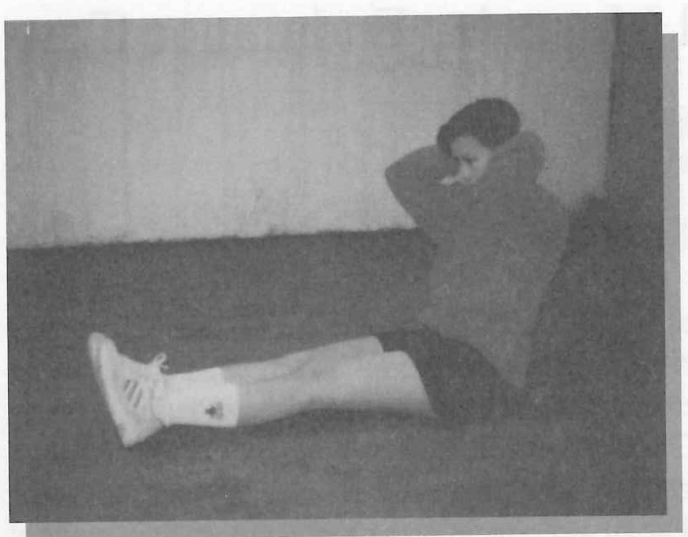

圖一: 直膝及雙手抱在頭後的仰臥起坐
作, 而且在這項測試中, 要求參與者把隻腳固定, 交叉貼 置於胸腹的雙手手肘部份在坐起時必須觸及大腿, 才算是 一次成功的坐起 (圖二)。像這樣的固定雙腳和全幅度的 動作要求, 一直受到質疑 (Liemohn, 1991)。

雖然雙腳在進行仰卧起坐時屈曲, 可減少璄屈肌群, 特 別是腰大肌 (Psoas Major) 的參與程度。從圖三可見, 腰大 肌的起點是腰椎的體部及橫突 (Transverse processes and bodies of lumber vertebrae)，止點是股骨的小轉子 (Lesser trochanter of femur) 。其主要作用是把腰椎向前彎曲, 屈 曲和向外旋轉大腿 (Tortora \& Grabowski, 1993) 。但當固 定雙腳進行仰臥起坐時, 腰大肌便會拉扯腰椎, 藉此幫助 腰腹靠近大腿。如果腹肌強壯, 借助腰大肌力量的程度也 減少。但如果腹肌力量及耐力不足, 或在重覆練習後出現疲勞, 例如一分鐘修改式仰卧起坐測試的最後十多 秒, 參與者腹肌耐力出現不足時, 腰大肌便會積極參與坐起的動作。這時候, 腰椎便被強烈拉扯而造成過度 前彎, 腰椎椎體間的壓力便大大增加, 問題嚴重的話, 下背痛便出現 (Jette, Sidney, \& Cicutti, 1984)。對於 一些本身腰背有毛病的人士，這種形式的仰卧起坐可能引發的問題便更多。

利雅蒙 (Liemohn, 1991) 更批評一分鐘曲滕仰卧起 坐測試法 (1-minute timed sit-up test, 跟修改式仰臥起 坐測試法相同) 中要求受試者作接近全幅度摺腹坐起動 作的做法。據 DeLacerda (1978) 指出在仰卧起坐動作 中, 腹肌最有效的參與範圍是由上背離開地面而屈起至 四十五度間的幅度。然而, 在一分鐘曲膝仰卧起坐測試 中受試者上身坐起的幅度平均為六十六度 (Barlow \& Neeres, 1978) 和七十五度 (Ricci et al., 1981)。研究亦 指出, 在四十五度以下的幅度, 參與屈腹坐起的肌群主 要是腹肌, 而脜屈肌則沒有參與 (Barlow \& Neeres, 1978; Ricci et al., 1981) 。但當上身屈起超過四十五度 時, 媳屈肌群, 包括髂肌 (Iliacus), 腰大肌和腰小肌 (Psoas Major \& Minor), 及股直肌 (Rectus Femoris) 都 會參與這個後半段的坐起動作。換句話説, 這種接近全 幅度的仰臥起坐測試, 不單止考驗受試者的腹肌耐力, 同時亦測驗其腟屈肌群的耐力。其中, 腰大肌的積極參 與, 更是增加腰椎前彎弧度和受傷機會的主要危機 (Sharpe, Liemohn, \& Snodgrass, 1988) 。而 Liemohn (1991) 更把這種固定雙腳及要求接近全幅度的仰臥起坐 列為具潛在危險的運動。

如果我們做仰卧起坐的目的是加強腹肌力量及耐力 的話, 當然希望能夠採用一個既安全, 又有效的鉎鍊方 法。然而, 在一分鐘曲膝仰臥起坐測試的成績和在等速 收縮 (Isokinetic contraction) 測量儀器所得的腹肌力量測 試數據之相關係數中, 發現無論在向心 (Concentric) 和 離心 (Eccentric) 的腹肌收縮上兩者的相關都很低。由於 等速收縮測量儀器的屈腹動作, 是針對腹肌力量的測 試。如果仰臥起坐所得的成績跟等速收縮測試所得的成 績成正比和高度相關的話, 也表示主要參與仰臥起坐動 作的是腹肌。但研究發現卻未如一般想像 (Hall, Hetzler, Perrin, \& Weltmen, 1992)。基於這些問題和證據, 更加 速了研究者和專家們要找出可以取代仰卧起坐的適當運 動。

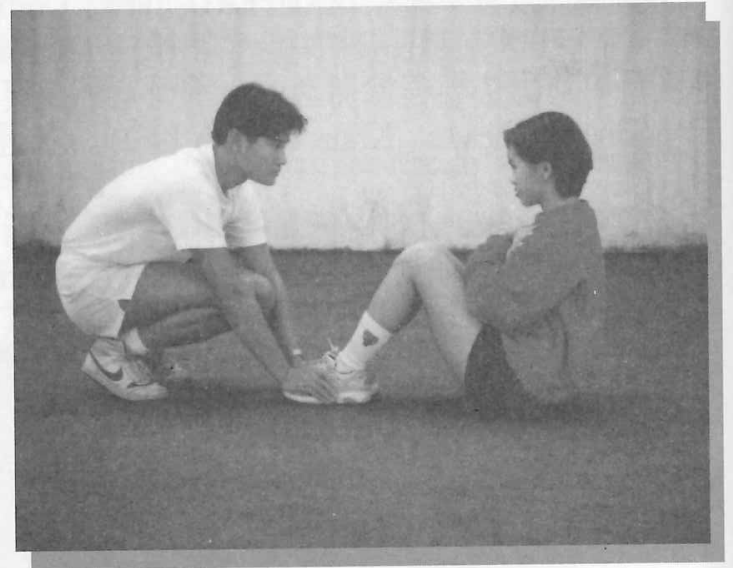

圖二 : 修改式仰臥起坐測試法

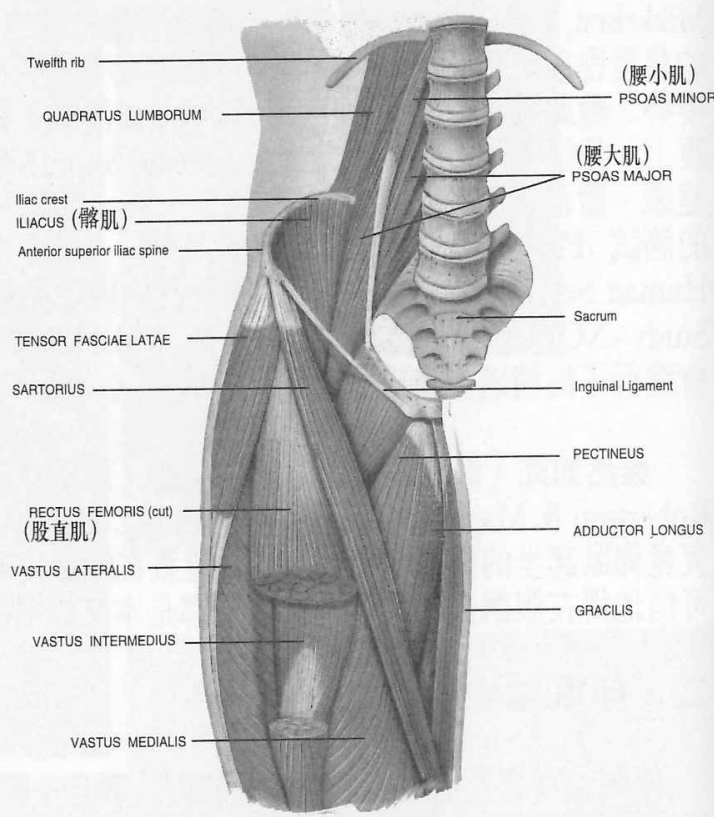

圖三: 璄屈肌群解剖圖 


\section{三－仰㤵港腹的好處在那裡？}

早在七十至八十年代初期，已有多位學者 (Gutin \& Lipetz, 1971；Kelley, 1982；Vincent \& Britten, 1980) 主張以仰臥捲腹 (Curl-up) 來代替仰卧起坐。當時, 最少亦有兩項有關仰臥捲腹測試的研究報告發表 (Noble, 1975; Vincent \& Britten, 1980) 。其中, Noble (1975) 研究計時和非計時的仰臥捲腹加轉腰 (Curl \& twist) 的動作, 姿勢是曲膝, 雙腳不固定, 雙手置於頭後。其中計時的一項測試, 更獲得相當高的信度 (reliability) (男 : $r=.91$; 女 : $r=.81$ )。

可惜, 當時仰臥捲腹仍未能受到重視，仰 臥起坐仍然大行其道。直至更多有關的研究公 佈後，情況才逐步改善。爭取採用仰臥捲腹來 代替仰臥起坐的聲音也愈來愈大。其實，仰臥 捲腹的動作形式 (圖四) 跟仰卧起坐的類似, 所不同者, 在於前者雙腳不被固定, 而且上身 屈起的角度不超過四十五度。這個動作也是針 對固定雙腳的仰卧起坐對腰椎構成壓力和傷害 的問題而作出修改。

羅拔臣和麥紐士托狄雅 (Robertson Magnusdottir, 1987) 進行了一項研究, 比較 仰臥捲腹和仰臥起坐兩者的分別, 當中研究者 發覺其中三位患有下背痛的受試者不能完成一 次的仰臥捲腹測試, 主要原因是由於下背問題 而削弱其屈腰動作的能力, 但其中一位卻能夠 完成十三次仰卧起坐測試。基於這點。研究者 認為她之所以能夠完成 13 次仰臥起坐, 是因 為借助其媳屈肌的力量。這結果更能証實仰臥 捲腹和仰卧起坐所要求的肌肉部份不同。對一 些腹肌較弱的人士, 在雙腳被固定的情況下, 往往能夠多做幾次仰卧起坐 (Ricci et al., 1981)。

透過表面肌電圖 (EMG) 研究, 進一步証實 仰臥捲腹動作是以腹肌為主, 而䂓屈肌沒有受 到刺激, 但在仰臥起坐動作中, 睍屈肌的參與 卻大於腹肌 (Robertson, Darville, \& Magnusdottir, 1986) 。在另一項研究仰臥捲腹 測詿的動作幅度中, 得出年齡平均 25 歲的受 試者, 其腰腹向上屈起的平均幅度為 $26.5^{\circ}$ (男, 15 人) 和 $37.9^{\circ}$ (女, 15 人)。兩者都 在有效幅度 $\left(45^{\circ}\right)$ 範圍內。而且遠較一分鐘 曲膝仰臥起坐測試動作所得的 $66^{\circ}$ (Barlow $\&$ Neeres, 1978) 和 $75^{\circ}$ (Ricci et al., 1981) 低。此外, 分別在仰臥捲腹和仰卧起坐兩種測 試所得成績的相關亦不高。進一步証明兩者的 差別 (Robertson \& Magnusdottir, 1987) 。從上 述多項証據顯示, 健體者的確有放棄仰臥起坐 而採用仰臥捲腹的必要。

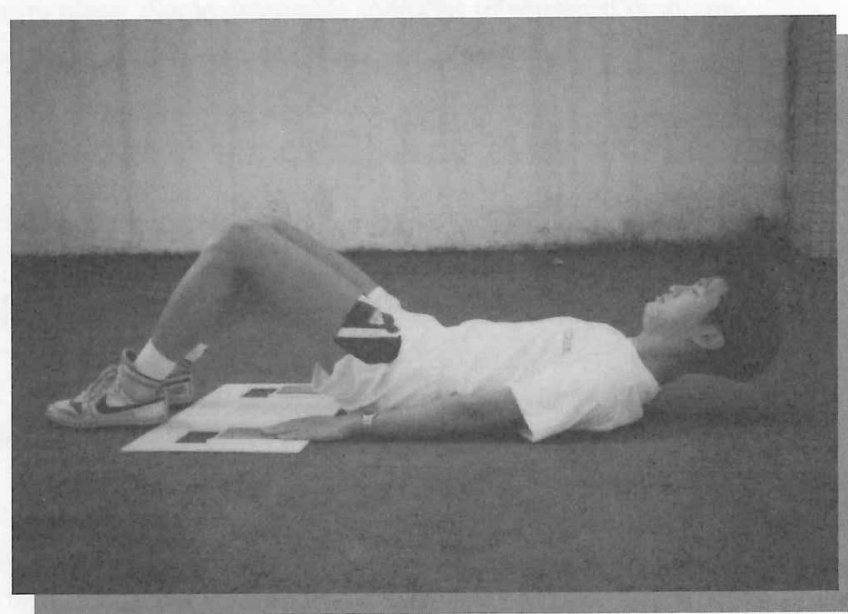

圖四一A: 仰卧捲腹測試法的開始姿勢

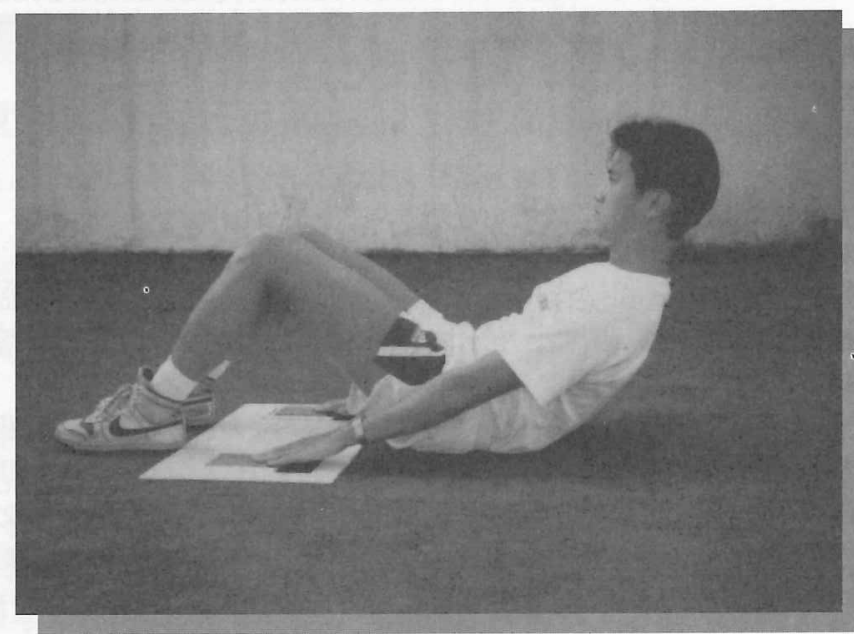

圖四一B：仰卧捲腹測計法的上屈完成姿勢

\section{四・仰卧輥腹測試}

為了更安全和有效的測試腹肌耐力，羅拔臣和麥紐士托狄雅 (Robertson \& Magnusdottir， 1987) 設計了 一套既易執行, 而且信度高 (男: $r=.93$; 女 : r=.94) 的仰臥捲腹测試法。一些學者為了表揚他們的成就, 更以 主要研究者的名字來命名, 稱為羅拢臣修改式仰臥捲腹測試法 (Robertson's Modified Curl-up Test)(Macfarlane, 1993) 。這項測試的姿勢要求和動作要點詳列於下： 


\section{(一) 婸地及器材}

1. 場地 :

測試場地冊須舖上軟熱，但表面不能太滑，以免受 試者在動作進行時慰部前後滑動（最好是躺在短毛 地毯上進行）。

2. 器材 :

在地面上貼上一條 3 吋 $(7.62 \mathrm{~cm})$ 闊的膠帶, 膠帶必 須牢固。此外，亦可利用專為仰臥捲腹測試而設的 3. 計時秒錶 仰臥捲腹架（圖五）。

\section{(二) 一般準則}

1 測試時間為一分鐘。

2 受試者進行動作時不應閉氣。

3 協助測試者數算時應大聲説出受試者成功的仰臥捲腹次數, 如果該次動作未能正確完成, 協助者必須重覆呼出同一累積 次數，籍此通知受試者作出正確的動作。

\section{(三) 動作要點}

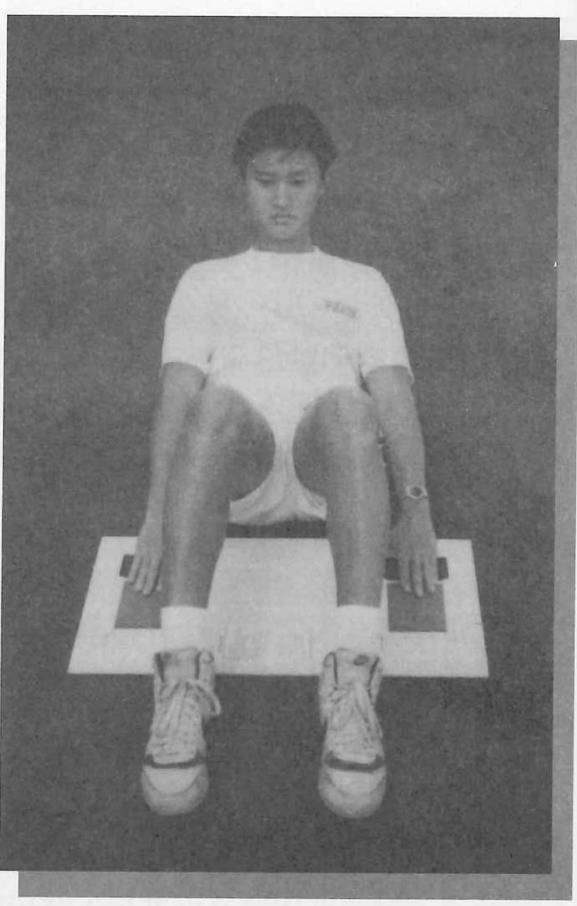

圖五：利用仰臥捲腹測試架進行測試的正面觀

1 受試者躺下, 曲膝約 $90^{\circ}$, 頭部放鬆。肩膊必須往足跟方向下壓, 下背全部份平放在地面（圖四 -A）。

2 肩膊下壓後, 雙手中指指尖剛好置於膠貼近端的邊緣, 如果利用仰臥捲腹架的話, 協助測試者需要把架 固定。

3 受試者准許有五次的練習, 以肯定動作是否正確。

4 開始測試後, 受試者的頭部可以保持離地。成功的一次仰臥捲腹動作是把上背屈起, 直至雙手中指指尖 同時滑過那 3 吋闊的膠貼後緣, 再把上身回復到上屈前姿勢, 直至其胛骨部份觸及地面為止。在整個動 作過程中, 受試者的慰部, 足跟, 及手指尖必須保持觸地, 如果未能符合上述任何一項, 該次動作便被 視為不正確及失敗。

5 在一分鐘內，受試者可以成功完成的捲起次數便是測試所得的成績。

6 測試完畢，受試者還原躺下，其雙手中指指尖的距離應該仍然在膠貼近端的邊緣，否則，受試者的動作 距離便有所改變，測試成績便不能準確。

7 測試完畢後，提醒受試者做些伸展和舒緩腹部肌肉的運動。

\section{五・結語}

從實際嘗試和操作中，筆者經驗到在起初階段，推行仰臥捲腹並不容易。一般健體者已習慣仰臥起坐的動 作模式，而主張幅度小及不固定雙腳進行的仰臥捲腹，常被誤為對於腹肌鉎鍊不足的運動。

在測試方面, 遇到的問題更多。如受試者在習慣仰臥起坐而又未能適應仰臥捲腹的情況下, 往往未能達到 測試的要求而完成更多的正確仰臥捲腹動作。其中, 最常見的不正確姿勢包括慰部移動、肩膊上下提壓、手 肘或手指做屈伸動作、借助挺腰來幫助屈腹的動作等。在多項可能影響測試準確度的因素, 以及受試者缺之 足夠腹肌力量和耐力的情況下, 測試者一時間亦難以獲得準確和理想的成績。雖然如此, 筆者相信這是個短 暫且必經的階段, 待健體者經過一段時間的練習, 以及掌握仰臥捲腹的動作要點後, 仰臥捲腹測試的效度 (Validity) 和信度 (Reliability) 必會大大提高。如果我們堅信健體運動可以增強體適能和為身心帶來健康的話, 我們必須肯定每項運動的安全和效果。因此, 我們還應留戀在仰臥起坐的日子嗎?

註：仰臥捲腹是筆者對 curl-up 的譯名，目的在於強調有效鍛鍊腹肌的捲腹動作。 


\section{參考文獻：}

AAHPERD. (1980). Health related physical fitness manual. Reston, VA: Author.

AAHPERD. (1988). Physical Best: A physical fitness education and assessment program. Reston, VA: Author.

Barlow, D. A., \& Neeves, R. E. (1978). Biomechnical assessment of partial iliopsoas isolation in women and its implications for athletic training. In F. Landry \& A. R. William (Eds.), Biomechanics of Sports and Kinanthropometry (pp. 53-59). Miami, Florida: Symposia Specialists.

Corbin, C. (1987). Physical fitness in the K-12 curriculum: Some defensible solutions to perennial problems. Journal of physical Education, Recreation, and Dance, 51(1), 49-55.

DeLacerda, F. (1978). Anatomical analysis of basic abdominal exercise. Journal of Physical Education, 75(5), 114-115.

Falls, H. B., Baylor, A. M., \& Dishman, R. K. (1980). Essentials of Fitness. Philadelphia, PA: Sounders College.

Flint, M., \& Gudgell, J. (1965). Electromyographic study of abdominal muscular activity during exercise. Research Quarterly, 36, 29-37.

Gutin, B., \& Lipetz, S. (1971). An electromyographic investigation of the rectus abdominis in abdominal exercises. Research Quarterly, 42, 256-263.

Hall, G. L., Hetzlar, R. K., Perrin, D., \& Weltmen, A. (1992). Relationship of timed sit-up tests to isokinetic and abdominal strength. Research Quarterly for Exercise and Sport, 63(1), 80-84.

Hong Kong Physical Fitness Association. (1993). Health related fitness \& lifestyle of working population aged 20-39 in Hong Kong. Hong Kong: Author.

Heyward, V. H. (1991). Advanced fitness assessment \& exercise prescription. Champaign, IL: Human Kinetics.

Jette, M., Sidney, K., \& Cicutti, N. (1984). A critical analysis of sit-ups: A case for partial curl as a test of abdominal muscular endurance. Canadian Alliance of Health, Physical Education and Recreation Journal, 51, 4-9.

Kelley, D. A. (1982). Exercise prescription and the kinesiological imperative. Journal of Physical Education, Recreation and Dance, 53(1), 18-20.

Kendall, F. P. (1965). A criticism of current tests and exercises for physical fitness. Physical Therapy, 45(3), 187-197.

Kraus, G. A., \& Raab, V. (1961). Hypokinetic disease. Springfield, IL: Charles C. Thomas.

Leimohn, W. (1991). Choosing the safe exercise. Certified News, 2, 1-3.

Macfarlane, P. A. (1993). Out with the sit-up, in with the curl-up! Journal of Physical Education,Recreation, and Dance, 64(8), 62-66.

Noble, L. (1975). A new curl-up test of abdominal endurance. in Abstracts of Research Papers 1975 AAHPER Convention (p. 115). Reston, VA: AAHPER Publications.

Plowman, S. A. (1992). Physical activity, physical fitness, and low back pain. In J. O. Holloszy (Ed.), Exercise and Sport Science Reviews, 20, 221-242. Baltimore, MD: Williams \& Wilkins.

Ricci, B., Marchetti, M., \& Figura, F. (1981). Biomechanics of sit-up exercises. Medicine and Science in Sports and Exercise, 13(1), 54-59.

Robertson, L. D., Darville, D., \& Magnusdottir, H. (1986). Abdominal fitness testing--a new approach. In T. Reilly, J. Watkins, \& J. Borms (Eds.), Proceedings of the VIII Commonwealth and International Conference on Sport, Physical Education, Dance, Recreation and Health, Kinanthropometry III, (pp. 227-232). London: E. \& F. N. Spon. 
Robertson, L. D., \& Magnusdottir, H. (1987). Evaluation of criteria associated with abdominal fitness testing. Research Quarterly for Exercise and Sport, 58(3), 355-359.

Seefeldt, V., \& Vogel, P. (1986). The value of physical activity. Reston, VA: AAHPERD.

Sharpe, G. L., Leimohn, W. P., \& Snodgrass, L. B. (1988). Exercise prescription and the low back--Kinesiological factors. Journal of Physical Education, Recreation \& Dance, 59(9), 74-78.

Sinaki, M., \& Mikkelsen, B. A. (1984). Post menopausal spinal osteoporosis: Flexion versus extension exercise. Archives of Physical Medicine and Rehabilitation, 65, 593-595.

Tortora, G. J., \& Grabowski, S. R. (1993). Principles of anatomy and physiology. New York: Harper Collins.

United States Department of Health and Human Services. (1985). Summary of findings from the National Children and Youth Fitness Study. Journal of Physical Education, Recreation, and Dance, 56(1), 43-90.

United States Department of Health and Human Services. (1987). Summary of findings from the National Children and Youth Fitness Study II. Journal of Physical Education, Recreation, and Dance, 58(9), 49-96.

Vincent, W. J., \& Britten, S. D. (1980). Evaluation of the curl-up as substitute for the bent-knee sit-up. Journal of Physical Education and Recreation, 51(2), 74-75.

Walters, C. E., \& Partridge, M. J. (1975). Electromyographic study of the differential action of the abdominal muscles during exercise. American Journal of Physical Medicine, 36, 259-268.

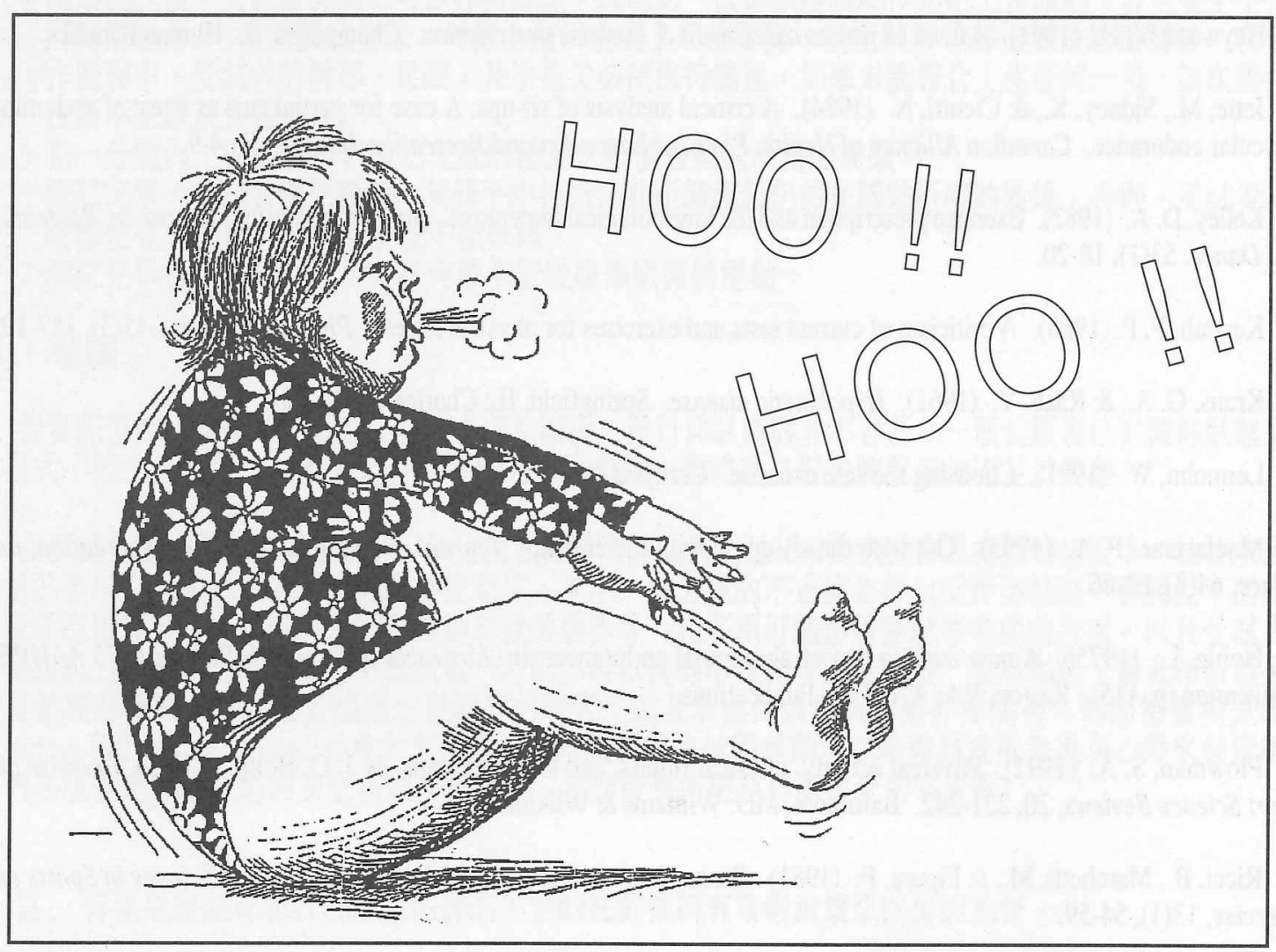

